\title{
5 \\ How can I, as an adult, facilitate play?
}

Nicole Bianquin ${ }^{1}$

\section{Adults have a crucial role in children's play}

The literature highlights the fact that children learn to play: they usually learn by themselves with self-initiated play activities or imitating others in inclusive play situations, but sometimes they need adult's support.

\section{Play for the sake of play can also be learnt.}

An educator, a therapist or a parent can take part of the child's play to improve, increase, and develop it. The role of the adult should be limited to pursuing objectives inherent to the play itself, not turning play into a play-like activity for reaching external goals, other than recreational pleasure and enjoyment.

This awareness is not yet clearly shared. Often adults (parents or professionals) exert too much control over the child's play. Reasons for that include: a) over-protection; b) giving priority to interventions with educational or therapeutic purposes; c) discomfort or disapproval of the children's play; d) reduced expectations on independent activity of the child, motivated for example by a perceived low initiative and/or long child's reaction times; and e) underestimation of the value of play, considered as a waste of time and unproductive activity. Taking part of a child's play should be about playing with the child, fully aware of one's own adulthood 
and educational competence, but maintaining and respecting the constraints and limitations of play itself and taking action to consolidate it, change it, and increase its complexity and flexibility as play, not as a means for learning or development.

When children with disabilities are involved, adult's intervention in play is even more important, as extensively shown by international research. Without adults' assistance, play often unfolds at lower levels for children with disabilities. However, with the careful and appropriate support from adults, play can move through naive play situations towards increasingly complex and challenging play situations. The adult's role in this process is of the utmost importance: parents, educators and therapists must learn how to teach children play. This involves a knowledge that is not innate nor embedded in the cultural repertoire. For example, adults must learn:

- How to teach children to use their body, with specific capabilities and limitations, in order to find pleasure and enjoyment in play;

- How to stimulate imagination, intellectual challenges, and relationships with others;

- How to set up the context to promote play (e.g. help the child to find a comfortable and functional play position, make the space welcoming and play appropriate, select the appropriate toys);

- How to apply specific play intervention techniques and methodologies; or

- How to use available advanced technology to facilitate and/or assess play.

The guiding principle is that any intervention to promote play must identify the child's characteristics and allow sufficient flexibility, unpredictability and safety for children to play. Adults' interventions should aim for creating an environment that supports children to choose freely what to do and how to do it. We hope that the present publication will guide an adult willing to acquire these competencies in his/ her journey. 


\section{Becoming a builder of rights}

All adults have responsibility for children's play, whether they are parents, educators, therapists, policy makers, or simply a fellow human being. Adults should be aware of the importance of play, play for the sake of play and not play with secondary goals, and promote and protect the conditions that support it. When interacting with children, adults should adopt a playful framework, both mentally and physically, provide dedicated time and space to play, and respect children's own play preferences, desires and needs. The adult in this context becomes a builder of rights. By granting the child the right to play, the main dimension of every child's life, he is recognising the right to childhood and the right to belong to a society that respects and welcomes the typical actions of children, and their contribution and approach to social life.

\section{Becoming a promoter of inclusive play}

Research studies show that children with disabilities have a desire to take part of the children's world. Playing with other children is the way to enter that world. The first challenge is to overcome social and attitudinal barriers. Adults need to understand the importance of play for every child and the benefits of an inclusive setting for all children. For example, a typically developing child, when used to model exemplary behaviours to his/her peers with disabilities, is likely to show increased self-esteem, confidence, autonomy and leadership skills. Adults should not overlook providing inclusive play opportunities to children with disabilities. These can happen indoor or outdoor, whenever more than one child meet together, for example in birthday parties, family reunions or an afternoon walk to the park. Inclusive play is instrumental to children with disabilities for making friends, experiencing childhood, and becoming a full member of the society. A child with enough inclusive play opportunities will learn how to manage conflicts and will be able to move between different social groups, expanding his/her capacity to communicate. Often adults are called to facilitate play, mediating interactions and constructing relationships between children, so they can learn to play together. They should however try to intervene as little as possible, allowing children to discover each other. 


\section{Becoming a scaffolder}

When facilitating play, the adult's goal should be to guide and support children with disabilities in a path that leads them to acquire the skills and abilities necessary to become an expert in play for the sake of play, creating the environmental conditions that are most suitable for the functioning of that child, overcoming architectural, social and personal barriers. In this role, an adult is a scaffolder, a person who erects scaffolding. Scaffolding is placed around the outside of buildings under construction allowing builders to access the emerging structure as it rises from the ground. Once the building is able to support itself, the builder removes the scaffolding.

The metaphor of scaffolding refers to the temporary support provided for learners to help them complete a task they would otherwise not be able to complete on their own. Effective scaffolding is tuned to the actual needs of learners, being constantly adjusted in response to their achievements, gradually fading away as they acquire the necessary skills.

Fundamental to the scaffolding methodology is the ability of identifying the child emotional and communication signals and appropriately respond to them, thus enabling a meaningful and effective interaction. The adult can assume three different main roles in facilitating and supporting play for children with disabilities: 1) Observer; 2) Activator; and 3) Partner. The adult will dynamically change his/her role and change the interaction with the child accordingly.

\section{Adult as Observer}

The first role of an adult when facilitating play is that of an Observer. The goal is to identify the child play skills and what he/she would like to do. It is essential to gather information about the child himself (e.g. desires, needs, degree of autonomy, attention, concentration) and about the child's play (e.g. play preferences, degree of playfulness, recurring or missing play typologies) in order to be able to build play situations that are appropriate for the particular child. In this process, the adult must be aware that his observations are always influenced by the models 
of play and of education that are present in his memory. It is therefore important to internalize the concept of play: 'a range of voluntary, intrinsically motivated activities normally associated with recreational pleasure and enjoyment'.

Although parents have an in-depth knowledge of their children, they should not neglect the need of purposely observing their children in order to understand how they play, with what, with whom, and also with what and with whom they would like to play. Professionals as well should take the time necessary to observe and get to know the child. Numerous tools are available today to support play observations, assessing for example the play autonomy, the levels of playfulness, or the play typologies (see Chapter 4 for more details).

\section{Adult as Activator}

The adult in the role of Activator should identify the best promising conditions from which play can spring, exist and evolve. A so-called playful framework should be created. Taking into consideration the child characteristics and having in mind that play is the foundation of childhood, the activator dedicates space and time for the child to experience play for the sake of play. Playful and inclusive play situations need to be designed and suitable toys need to be chosen. The activator role involves several tasks, including:

- Organising or selecting play environments;

- Making time for play in the child and adult daily routines;

- Choosing and making available to the child appropriate toys;

- Planning and suggesting play activities that interest, intrigue and motivate the child;

- Fostering inclusion, e.g. creating play situations that naturally result in physical contact between children with and without disabilities. 


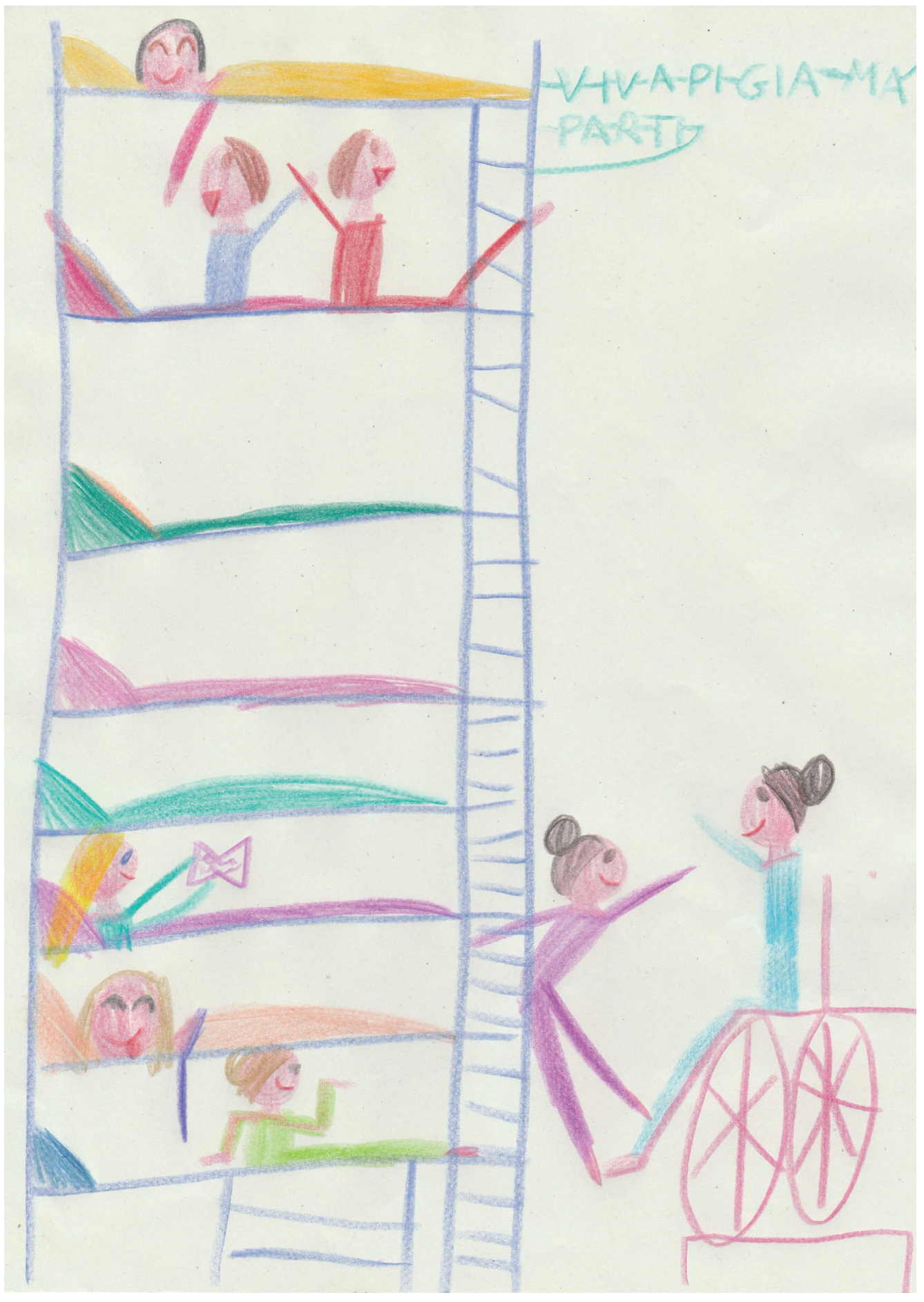

Arianna, 7 years old, Italy 


\section{Adult as Partner}

The role of Partner is the closest to that of a scaffolder. The adult becomes a play partner, whenever possible following and not directing the child. He/she should keep in mind that the sole purpose of play is recreational pleasure and enjoyment; it is not an opportunity for education or therapy. Being a play partner implies sharing the meaning and purpose of the activity, losing the typical adult/child asymmetry, becoming immersed in reciprocity and complicity, allowing the child to express his/her full potential. Interventions should be limited to suggest changes, feasible to the child, with the purpose of expanding, varying and/or enriching the child's play interests, always within the play framework. Knowledge on developmental models of play may help the adult to sustain the child's action, to 'work with and through play' taking into consideration the child's characteristics, mechanisms, rhythms, times and needs. Children with different types of disability may require different actions from the adult so he/she succeeds in becoming a play partner. Some examples are listed below, according to the type of disability:

- Children with Intellectual Disabilities - By creating an emotionally stable environment and with the proper support, children with intellectual impairments should be able to demonstrate a certain level of autonomy in play. Physical guidance; modelling, showing concretely how to do things and how to use objects; and repetition may be useful techniques. Adults should always be kind, patient and enthusiastic playmates, respecting the child rhythms, sharing roles and rules, and making sure that the child is having fun.

- Children with Hearing Impairments - Children with hearing impairments may need support in understanding the play situation, in expressing their needs and desires, and in acquiring some degree of autonomy. The adult should carefully choose an appropriate relative position with respect to the child during play. For example, sitting face-to-face and close to the child, promoting visual and physical interactions, showing the toys and inviting the child to play, may help the child to overcome the barriers and actively engage in play. The adult should also serve as a facilitator of inclusion, inviting other children to play. Whenever children start playing with each other, the adult should retreat to the role of an observer, reducing interventions to the minimum possible.

- Children with Visual Impairments - In order to support children with visual impairments, adults can assume the role of a playmate that is always talking about what is happening, thus providing the necessary feedback on the play 
situation to the child. For example, in case of constructive play, frequent feedback on how construction is going can help the child to mentally represent the object being built and plan the necessary actions to finalize the project. When other children are involved, adults may mediate the relationships between the child with visual impairments and other children, thus ensuring that all children actively take part of play.

- Children with Communication Disorders - The essential task of adults, when playing with children with communication disorders, is to support and enrich children's language, enabling them to express their feelings, ideas, needs and requests. Enough time should be given for the child to convey his/her message, without constantly trying to guess what the child has to say. In addition, adults have a central role in promoting and facilitating inclusive play. They should help the child with communication disorders to understand the play situation and the playmates' proposals, but also help the other children in learning how to communicate with the child with communication disorders. The ultimate goal is to develop in all playmates the necessary communication skills such that play can happen without adult intervention.

- Children with Severe Physical Impairments - The adult playmate of a child with severe physical impairments should be very patient, allowing the necessary time for the child to complete an action by him/herself, resisting to the urge of substituting the child. The adult should provide opportunities for the child to play autonomously, regardless of his/her impairment, for example making use of adapted toys. In inclusive play situations, the adult should also pay attention to the other children, helping them in finding appropriate ways of interacting with the child with physical impairments, and promoting play activities in which all can take an active role.

- Children with Autism Spectrum Disorders - When playing with a child with Autism Spectrum Disorders, the adult needs to gain the trust of the child and design a play framework in which the child feels comfortable and safe. Adults should then gradually introduce novelties into the play situation. For example, one can take a story that is already familiar to the child and invite the child to play a role in it, then introducing some new elements. In the case of constructive play, the adult may model how to go on with the construction, preventing the child from always focusing on the same sequence. Special attention should be given to avoid or reduce frustrations, clearly explaining the play situations and providing support when needed. Additionally, adults should ensure that the 
sensorial characteristics of the play situation (lights, colours, sounds, objects, smells) are appropriate for the child.

- Children with Multiple Disabilities - Although sometimes extremely challenging, play is as fundamental for children with multiple disabilities as for any other child. Physical contact and continuous verbalization should be used to patiently explain, give meaning, encourage, and congratulate the child. Especially with these children, adults should build on their interests and preferences, proposing play activities that are of their interest and that they are able to perform, and then slowly increase the challenge to the child. Often play with these children involves repeated patterns of movement or sound (practice play) and only with the proper support from an adult it can evolve in variety and complexity.

\section{Conclusion}

Adults have a crucial role in children with disabilities' play. By clearly understanding the importance of play for the sake of play for all children, adults can become guardians of the children's right to play, ensuring them enough opportunities for playing by themselves and with others. In play situations, adults are called to support children. They should observe to understand the child play skills, preferences and needs; then they should activate play by creating a motivating play framework; finally, they should become play partners, always respecting the child's play preferences, providing only the needed support, and making sure that the play always contains the right amount of challenge to keep the child engaged. The metaphor of scaffolding is particularly enlightening: the adult should support the child while he/she is acquiring play skills in the same way scaffolding supports the construction of a new building. Once the child has acquired the necessary skills to play on his/her own, scaffolding should be removed. 